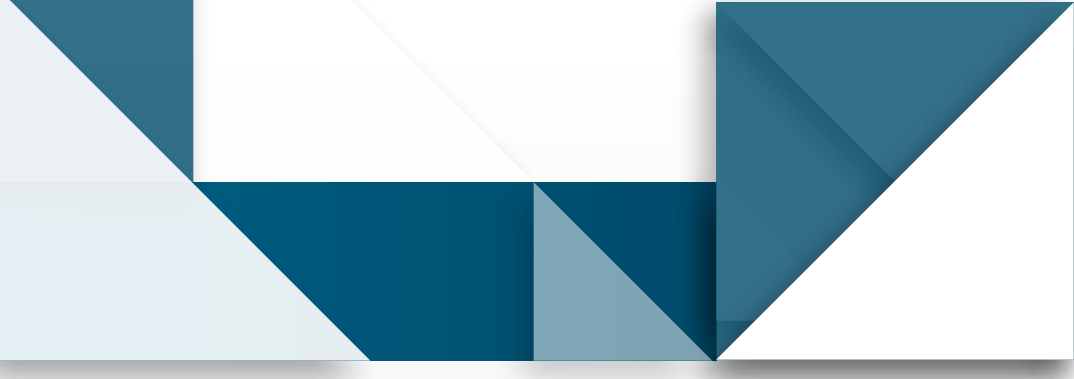

\title{
Estudio transversal de las 3 actitudes hacia la ciencia en estudiantes de grados quinto a undécimo. Adaptación y aplicación de un instrumento para valorar actitudes
}

- Cross-sectional study on secondary students' attitudes towards science. Adaptation and application of an attitude questionnaire

- Estudo transversal das atitudes em relação à ciência de estudantes do $5^{\circ}$ ano do Ensino Fundamental e $3^{\circ}$ ano do Ensino Médio: Adaptação e aplicação de um instrumento de atitudes

\section{Resumen}

El presente trabajo muestra el estudio de las actitudes hacia la ciencia en un grupo de estudiantes a lo largo de los diferentes niveles de secundaria, según la implementación, ajuste y aplicación de un instrumento evaluativo propuesto originalmente por Barmby, Kind y Jones (2008). La aplicación del instrumento y de una entrevista semiestructurada permitieron obtener resultados confiables (alfa de Cronbach, $\alpha=0,77$ )sobre diferentes categorías de actitud frente a la ciencia. Los resultados indican que, en la población estudiada, no hay diferencias significativas respecto al género. Se observaron concepciones favorables respecto a la importancia de la ciencia y la tecnología y al trabajo práctico en ciencias, pero desfavorables frente a las

\section{Palabras clave:}

Actitudes, ciencia, actitudes hacia la ciencia, educación secundaria, enseñanza.

Manuel Molina* José Carriazo** Jaime Casas ${ }^{* * *}$

\footnotetext{
Universidad Nacional de Colombia: mfmolinac@unal.edu.co

** Universidad Nacional de Colombia: jcarriazog@unal.edu.co

*** Universidad Pedagógica Nacional: jcasas@pedagogica.edu.co
} posibilidades futuras de trabajar en el ámbito científico y con manifestaciones recurrentes sobre imaginarios de complejidad hacia la actividad científica. Finalmente, es evidente una disminución progresiva (desde grado quinto hasta grado undécimo) de las actitudes favorables frente a las ciencias. 


\section{Abstract}

This paper presents a study on secondary students' attitudes towards science. It was carried out by designing, adjusting and applying an assessment instrument initially designed by Barmby, Kind and Jones, (2008). The application of this instrument and a pedagogical semi-structured interview allowed the collecting of reliable results (Cronbach alpha, $\alpha=0,77$ ), using several attitude categories (attitude components or constructs) for science. According to the obtained data, there are not important differences regarding the gender (male or female students' attitudes). It was found that there are positive conceptions regarding the "importance of science and technology" and the importance of laboratory work in science". However, it was found that the students present unfavourable conceptions regarding the future possibilities of working in the science field. In addition, several recurrent ideas considering the science as a complex activity were observed. Finally, a progressive decrease of the positive attitudes to science was evident from 5 th to 11 th grade students.

\section{Resumo}

O presente trabalho mostra um estudo das atitudes em relação à ciência em um grupo de estudantes ao longo de diferentes níveis do Ensino Básico, por meio da implementação, ajuste e aplicação de um instrumento avaliativo proposto originalmente por Barmby, Kind e Jones (2008). A aplicação do instrumento e de uma entrevista semi-estruturada permitiram obter resultados confiáveis (alfa de Cronbach, $a=0,77$ ) sobre diferentes categorias de atitudes frente à ciência. Os resultados indicam que, na população estudada, não há diferenças significativas em relação a gênero. Se observaram concepções favoráveis a respeito de "a importância da ciência e da tecnologia" e do "trabalho prático em ciências", mas desfavoráveis diante das possibilidades futuras de trabalhar no âmbito científico e com manifestações recorrentes sobre imaginários de complexidade em relação à atividade científica. Por fim, é evidente a diminuição progressiva (desde o quinto ano até o terceiro ano do Ensino Médio) das atitudes favoráveis com relação às ciências.

\section{Keywords:}

Attitudes, science, attitudes towards science, secondary school, teaching.

\section{Palavras-chave:} Atitudes, atitudes em relação à ciência, educação básica, ensino. 


\section{Introducción}

Las actitudes del estudiante frente a cada área de estudio marcan un importante punto de partida en el desarrollo de su etapa escolar (Fensham, 2004). En particular, las actitudes hacia las ciencias están estrechamente ligadas con los logros académicos (Kurbanoglu y Akim, 2010 y Cukrowska, Staskun y Schoeman, 1999), y el desarrollo de actitudes positivas en los estudiantes hacia las disciplinas científicas constituye una de las grandes responsabilidades de cada profesor de ciencias (Cheung, 2009). Algunos investigadores, como Cukrowska, Staskun y Schoeman (1999) y Freedman (1997), encuentran que una buena actitud es correspondiente con un mejor desempeño académico. Por ello, en este momento de especial atención hacia la calidad de la educación, las actitudes que poseen los estudiantes hacia las diferentes áreas del conocimiento se convierten en parte esencial para mejorar los procesos de enseñanza y aprendizaje en diversas instituciones del mundo (Wang y Berlin, 2010, Kaya, Yager y Dogan, 2009, Kidman, 2009 y Reid, 2006). Así, esas actitudes son parte del reto de los docentes de ciencias, cuando se piensa no solo en formar científicos (ciencia propedéutica), sino en formar ciudadanos alfabetizados científica y tecnológicamente. La ciencia y la tecnología representan hoy en día un componente importante en el desarrollo de la sociedad, especialmente por el uso creciente de productos llamados tecnológicos, para lo cual, como consumidores de facto, sería favorable conocer ciertos contenidos básicos de la ciencia que permitan establecer criterios apropiados para el consumo responsable frente a las necesidades de la sociedad y el ambiente. Adicionalmente, para cualquier nación es importante trazar políticas que superen el consumo y conduzcan a sus ciudadanos a la producción de ciencia y tecnología o a su implementación en procesos productivos, lo que da mayor sentido y preponderancia a la formación de ciudadanos que posean un vocabulario científico y habilidades científicas muy generales, para que puedan involucrarse en el desarrollo de sociedades tecnificadas. En Colombia, la ciencia y la tecnología poseen un imaginario socialmente importante, como lo muestra un estudio reciente realizado por Colciencias (Aguirre, 2005), según el cual, la ciencia es vista como algo útil y cercana a la población, particularmente en el campo de la salud, asignándole mayor importancia a los beneficios que aporta que a los perivicios. Sin embargo, aunque se les ha dado importancia, la ciencia y la tecnologías e siguen percibiendo por el grueso de la población como una serie de actividades especiales realizadas por los países desarrollados. Al respecto, el estudio anterior concluye que:

A pesar de la actitud favorable hacia la ciencia y la tecnología, la percepción mayoritaria de ellas se caracteriza en este estudio por su escasa magnitud, quizás porque no se han dado los suficientes elementos para construir y comprender los acontecimientos, en donde la mirada sigue puesta en los resultados, mas no en los procesos que dan lugar a esos resultados. En este sentido, la ciencia como actividad social es sustituida por el imaginario de la tecnología (avances técnicos para el desarrollo de la sociedad), privándola de sus finalidades iniciales, las que reflejan los puntos de vista y los valores de la sociedad, en donde el consumo de información científica ofrecido a través de los medios de comunicación incide en la capacidad de la gente de formarse un imaginario social sobre este tema. (Aguirre, 2005. pp. 18)

La actitud favorable que posee la ciudadanía sobre la ciencia no siempre se refleja en la escuela, máxime si se entiende que la ciencia que se desarrolla en el aula no necesariamente 
coincide con la que se percibe fuera de ella. Por ello, en el presente estudio damos cuenta de las actitudes hacia la ciencia escolar, es decir, la que se desarrolla dentro del aula.

Por otra parte, los estudios sobre actitudes hacia la ciencia permiten tener información para diseñar nuevos currículos y para entender el trabajo del profesor, así como el resultado de sus esfuerzos y estrategias. La mayoría de estudios actitudinales corresponden a estudios puntuales, pero pocos a estudios a lo largo de la etapa escolar (Tekbiyik y Akdeniz, 2010, Wang y Berlin, 2010, Cheung, 2009, Vázquez y Manassero, 2008 y Salta y Tzougraki, 2004), y mucho menos a nivel local en Colombia. Las investigaciones muestran que existen dos resultados recurrentes sobre actitudes hacia la ciencia: el declive de las actitudes hacia la ciencia escolar al acercarse a grados superiores y las diferencias relevantes entre las actitudes de niños y niñas, (Hofstein y Mamlok, 201 1, Cheung, 2009 y Vázquez y Manassero, 2008). Aunque estos resultados son comunes en diversos estudios, estos no son comparables; así, por ejemplo, al utilizar una escala numérica de actitud de 1 a 5, un valor de 3,5 en un lugar no puede afirmarse que sea una mejor actitud que la correspondiente a un 3,3 en otro. Adicionalmente, referente a la temática en estudio, Prieto y Vera (2008) aplicaron un protocolo de actitudes hacia la ciencia (PAC) a un grupo de 908 estudiantes de Bogotá (Colombia), encontrando que no hay diferencias significativas según el grado escolar y el género, pero sí diferencias significativas con respecto a la jornada escolar (mañana o tarde). Aunque el estudio es completo en su aplicación, los tratamientos estadísticos hacen difícil su reproducción por profesores no expertos; igualmente, no se presenta un análisis profundo de los resultados debido a que los autores dedican especial énfasis en el tratamiento estadístico. Si bien es importante soportar los estudios, muchos se apoyan más en la estadística que en la indagación sobre las variables que afectan los resultados. Por ello, en el presente estudio se ofrece un equilibrio en este sentido: se presta especial atención a los resultados, sin descuidar la rigurosidad del tratamiento estadístico asequible a profesores con conocimientos básicos en estadística.

En la misma dirección, existen estudios que muestran que en la etapa de transición escolar se encuentran cambios notables en las actitudes hacia la ciencia (Murphy y Beggs, 2006); sin embargo, no existen muchos resultados detallados al respecto. Por otra parte, en la disminución de las actitudes favorables hacia la ciencia, las mujeres presentan el mayor declive en varios estudios (George, 2006), debido quizá al ambiente escolar y posiblemente por influencias motivacionales por parte del profesorado (Mirandes, Sanmartí y Tarín, 1993). Algunos estudios muestran que, en el paso de los estudiantes de la primaria a la secundaria, el agrado por la ciencia empieza a declinar progresivamente hasta finalizar los estudios (Pell y Jarvis, 2001). Como se encontró en el estudio ROSE (Schreiner y Sjøberg, 2004), la disminución en las actitudes hacia la ciencia ocurre de forma más importante en los países llamados desarrollados, afectado por diversas variables como el sexo, la disciplina científica, la cultura, el colegio, 
los problemas sociales, el currículo y la forma como se desarrollan las clases.

Para contribuir con el conocimiento y la comprensión de esta problemática, en el presente trabajo se muestran los resultados de una investigación sobre las actitudes hacia la ciencia que posee un grupo de estudiantes de secundaria, las cuales se constituyen en aspecto relevante en la selección de carreras y en el papel que asumirán como ciudadanos a futuro, así como en el aprendizaje de la ciencia y en la apropiación de la ciencia como herramienta útil en la solución de problemas sociales.

\section{De las actitudes}

Para este trabajo, se asume la definición de actitud dada por Kind, Jones y Barmby (2007): "sentimientos que una persona tiene hacia un objeto basadoen su conocimiento y creencias acerca del objeto". En esta definición, los autores tienen en cuenta los componentes cognitivo, afectivo y comportamental del sujeto. Para el presente estudio, el objeto de actitud es la ciencia, así como los conocimientos y las creencias que tienen que ver con la ciencia de la escuela, el conocimiento cotidiano y los diferentes factores que influyen en el aprendizaje (grado, ambiente, profesores, libros, currículo, etc.). Las actitudes hacia la ciencia incluyen varias dimensiones, las cuales se encuentran reflejadas en el instrumento evaluativo, constituyen elementos esenciales para la valoración de actitudes hacia la ciencia y ya han sido empleadas en estudios anteriores (Barmby, Kind y Jones, 2008 y Kind, Jones y Barmby, 2007):

- Aprendizaje de la ciencia en la escuela.

- Autoconcepto de ciencia.

- Trabajo práctico en ciencia.

- Importancia de la ciencia.

- Ciencia fuera de la escuela.

- Futura participación en ciencia.
En el presente escrito, se muestran los resultados del estudio de las actitudes que poseen y desarrollan los estudiantes desde grado quinto hasta grado undécimo en un colegio oficial en Bogotá (Colombia), partiendo de la validación psicométrica de un instrumento adaptado de Barmby, Kind y Jones (2008). El estudio hace este recorrido para establecer la progresión respecto al nivel escolar como uno de los parámetros de influencia en los procesos de enseñanza y aprendizaje de la ciencia, a lo largo del ciclo educativo, y poder contrastar los resultados con otros estudios, como los de Vázquez y Manassero (2008) o Barmby, Kind y Jones (2008), quienes muestran un declive progresivo a lo largo de la educación secundaria obligatoria.

\section{Metodología}

El trabajo comprendió dos fases:

- Adaptación del instrumento y análisis psicométrico.

- Aplicación del instrumento y análisis de los resultados, sobre el instrumento afinado.

\section{Población y muestra}

Los datos presentados fueron extraídos de una población de 238 estudiantes (127 hombres y 111 mujeres) de quinto a undécimo grado de la Institución Educativa Distrital Restrepo Millán (Bogotá, Colombia) (tabla 1). Se seleccionó un curso de cada grado para aplicar el instrumento, con la colaboración de los diferentes profesores presentes en cada curso. Los estudiantes contaron con treinta minutos para responder. En los cursos inferiores, fue necesario discutir el ejemplo del cuestionario (anexo 1) y otros más para evitar confusiones en las respuestas entregadas. Las edades de los participantes van desde los 10 a los 18 
años y en todos los casos hubo total disposición para responder el cuestionario. Posterior a la aplicación del instrumento, fueron seleccionados de tres a cinco estudiantes de ambos géneros por cada curso para realizar una actividad experimental con los investigadores. Este grupo focal (28 estudiantes) fue conducido a un salón, donde se realizaron tres experimentos demostrativos, como una forma de estimular el dialogo alrededor de las preguntas presentes en el cuestionario. La entrevista con este grupo focal tuvo una duración de cuarenta minutos.

Tabla 1. Distribución de la población

\begin{tabular}{lcccccccc}
\hline Género/grado & Quinto & Sexto & Séptimo & Octavo & Noveno & Décimo & Undécimo & Total \\
\hline Hombres & 20 & 14 & 17 & 20 & 21 & 13 & 22 & 127 \\
\hline Mujeres & 14 & 18 & 13 & 17 & 15 & 20 & 14 & 111 \\
\hline
\end{tabular}

\section{El instrumento}

El instrumento diseñado consistió en un cuestionario adaptado a partir de uno desarrollado por Barmby, Kind y Jones (2008), el cual consta de seis categorías de actitudes. Adicional a esto, se incluyó la pertenencia a la institución educativa, para relacionar los resultados de las actitudes con el ambiente de la institución escolar. El cuestionario se muestra en el anexo 1 y a continuación aparecen los ítems que corresponden a cada categoría:

- Aprendizaje de la ciencia en la escuela: ítems 1, 2, 3, 4, 5 y 6.

- Trabajo práctico en ciencia: ítems 13, 14, 15, 16, 17, 18, 19 y 20.

- Ciencia fuera de la escuela: ítems 21, 22, 23, 24, 25 y 26.

- Importancia de la ciencia: ítems 32, 33, 34, 35, 36 y 37.

- $\quad$ Autoconcepto de ciencia: ítems 7, 8, 9, 10, 11 y 12.

- Futura participación en ciencia: ítems 27, 28, 29, 30, 31.

- Pertenencia a la institución educativa: ítems 38, 39, 40, 41, 42, 43, 44 y 45.

\section{Adaptación}

Se realizó siguiendo las consideraciones de Hambleton (2002), que se resumen a continuación:

- Traducción del cuestionario teniendo en cuenta el idioma y la cultura. Esta traducción se realizó por expertos en el área de las ciencias y en el idioma.

- Revisión de la versión adaptada del cuestionario por cinco expertos en investigación didáctica.

- Estudio de la consistencia interna de la prueba, tomando como referencia el alfa de Cronbach. 
- Aplicación de la prueba y análisis de los resultados. El cuestionario del anexo 1 constó de 45 preguntas distribuidas en orden para cada categoría, en el que tres de las preguntas están redactadas de forma negativa (6, 7 y 20).

\section{Resultados y discusión}

Luego de aplicar las encuestas, se realizó el procesamiento manual, para determinar la cantidad de respuestas a cada opción, y se transfirió esta información a una hoja de cálculo del programa Excel para realizar los tratamientos estadísticos correspondientes, como se muestra en el anexo 2 y en la tabla 2 (para una muestra de hombres). En cada ítem, para las cinco opciones de la escala de Likert se obtuvo una valoración; por ejemplo, en el ítem 1 (tabla 2) (en las clases de ciencias aprendemos cosas interesantes), trece alumnos seleccionan la opción totalmente de acuerdo (TA), ocho, de acuerdo (A) y cero, el resto de opciones. Los resultados obtenidos, sin referencia al tratamiento estadístico, indican que existe una actitud muy positiva hacia lo que se aprende en la clase de ciencias; sin embargo, para comprender mejor todos los resultados, se requiere la misma escala, que, como ya se expresó, fue seleccionada de 1 a 5 . Para convertir los valores a la escala adoptada (Likert) se multiplica la cantidad de opciones seleccionadas por el valor asignado a cada opción, en este caso, $13 \times 5$ y $8 \times 4$ (los resultados aparecen como valor en la tabla 2), se suman y se dividen por 21 (cantidad de alumnos que seleccionaron esas opciones de respuesta) para obtener 4,6 de promedio (tabla 2), corroborando una actitud muy positiva hacia ese ítem.

Tabla 2. Resultados sobre las actitudes para 21 estudiantes hombres de noveno y los promedios según el género (Hombre: H; Mujer: M)

\begin{tabular}{|c|c|c|c|c|c|c|c|c|c|c|c|c|c|}
\hline Ítem & TA & A & I & D & TD & & & Valor & & & Promedio & $\mathrm{H}$ & M \\
\hline 1 & 13 & 8 & 0 & 0 & 0 & 65 & 32 & 0 & 0 & 0 & 4,6 & 4,5 & 4,6 \\
\hline 2 & 2 & 9 & 6 & 4 & 0 & 10 & 36 & 18 & 8 & 0 & 3,4 & 3,5 & 3,6 \\
\hline 3 & 8 & 13 & 0 & 0 & 0 & 40 & 52 & 0 & 0 & 0 & 4,4 & 4,3 & 4,5 \\
\hline 4 & 4 & 5 & 9 & 2 & 1 & 20 & 20 & 27 & 4 & 1 & 3,4 & 3,4 & 3,6 \\
\hline 5 & 5 & 7 & 4 & 3 & 2 & 25 & 28 & 12 & 6 & 2 & 3,5 & 3,3 & 3,4 \\
\hline 6 & 0 & 1 & 0 & 11 & 9 & 0 & 2 & 0 & 44 & 45 & 4,3 & 4,1 & 4,3 \\
\hline 7 & 0 & 3 & 5 & 10 & 3 & 0 & 6 & 15 & 40 & 15 & 3,6 & 3,4 & 3,6 \\
\hline 8 & 2 & 14 & 5 & 0 & 0 & 10 & 56 & 15 & 0 & 0 & 3,9 & 3,7 & 3,6 \\
\hline 9 & 3 & 15 & 3 & 0 & 0 & 15 & 60 & 9 & 0 & 0 & 4,0 & 4,0 & 4,0 \\
\hline 10 & 7 & 8 & 6 & 0 & 0 & 35 & 32 & 18 & 0 & 0 & 4,0 & 3,8 & 3,8 \\
\hline 11 & 1 & 8 & 6 & 4 & 2 & 5 & 32 & 18 & 8 & 2 & 3,1 & 3,1 & 3,4 \\
\hline 12 & 5 & 11 & 5 & 0 & 0 & 25 & 44 & 15 & 0 & 0 & 4,0 & 3,7 & 3,8 \\
\hline 13 & 14 & 4 & 3 & 0 & 0 & 70 & 16 & 9 & 0 & 0 & 4,5 & 4,1 & 4,4 \\
\hline 14 & 6 & 14 & 1 & 0 & 0 & 30 & 56 & 3 & 0 & 0 & 4,2 & 4,1 & 4,4 \\
\hline 15 & 10 & 10 & 1 & 0 & 0 & 50 & 40 & 3 & 0 & 0 & 4,4 & 4,2 & 4,2 \\
\hline 16 & 5 & 7 & 7 & 2 & 0 & 25 & 28 & 21 & 4 & 0 & 3,7 & 3,8 & 4,1 \\
\hline
\end{tabular}




\begin{tabular}{|c|c|c|c|c|c|c|c|c|c|c|c|c|c|}
\hline 17 & 12 & 6 & 3 & 0 & 0 & 60 & 24 & 9 & 0 & 0 & 4,4 & 4,3 & 4,2 \\
\hline 18 & 13 & 6 & 2 & 0 & 0 & 65 & 24 & 6 & 0 & 0 & 4,5 & 4,4 & 4,5 \\
\hline 19 & 9 & 10 & 2 & 0 & 0 & 45 & 40 & 6 & 0 & 0 & 4,3 & 4,1 & 4,1 \\
\hline 20 & 0 & 0 & 0 & 5 & 16 & 0 & 0 & 0 & 20 & 80 & 4,8 & 4,2 & 4,2 \\
\hline 21 & 2 & 2 & 11 & 4 & 2 & 10 & 8 & 33 & 8 & 2 & 2,9 & 2,9 & 3,4 \\
\hline 22 & 5 & 9 & 2 & 3 & 2 & 25 & 36 & 6 & 6 & 2 & 3,6 & 3,5 & 4,0 \\
\hline 23 & 10 & 6 & 5 & 0 & 0 & 50 & 24 & 15 & 0 & 0 & 4,2 & 4,2 & 4,5 \\
\hline 24 & 8 & 6 & 3 & 4 & 0 & 40 & 24 & 9 & 8 & 0 & 3,9 & 4,0 & 4,1 \\
\hline 25 & 2 & 6 & 5 & 6 & 2 & 10 & 24 & 15 & 12 & 2 & 3,0 & 3,2 & 3,4 \\
\hline 26 & 9 & 9 & 3 & 0 & 0 & 45 & 36 & 9 & 0 & 0 & 4,3 & 4,2 & 4,3 \\
\hline 27 & 4 & 6 & 6 & 5 & 0 & 20 & 24 & 18 & 10 & 0 & 3,4 & 3,4 & 3,6 \\
\hline 28 & 4 & 5 & 6 & 4 & 2 & 20 & 20 & 18 & 8 & 2 & 3,2 & 3,2 & 3,4 \\
\hline 29 & 5 & 8 & 6 & 2 & 0 & 25 & 32 & 18 & 4 & 0 & 3,8 & 3,3 & 3,6 \\
\hline 30 & 1 & 1 & 3 & 8 & 8 & 5 & 4 & 9 & 16 & 8 & 2,0 & 2,6 & 3,0 \\
\hline 31 & 1 & 8 & 6 & 3 & 3 & 5 & 32 & 18 & 6 & 3 & 3,0 & 3,0 & 3,1 \\
\hline 32 & 18 & 3 & 0 & 0 & 0 & 90 & 12 & 0 & 0 & 0 & 4,9 & 4,7 & 4,7 \\
\hline 33 & 11 & 8 & 2 & 0 & 0 & 55 & 32 & 6 & 0 & 0 & 4,4 & 4,2 & 4,1 \\
\hline 34 & 2 & 10 & 6 & 3 & 0 & 10 & 40 & 18 & 6 & 0 & 3,5 & 3,5 & 3,5 \\
\hline 35 & 2 & 3 & 11 & 3 & 2 & 10 & 12 & 33 & 6 & 2 & 3,0 & 3,1 & 3,2 \\
\hline 36 & 8 & 12 & 1 & 0 & 0 & 40 & 48 & 3 & 0 & 0 & 4,3 & 4,2 & 4,5 \\
\hline 37 & 5 & 7 & 7 & 2 & 0 & 25 & 28 & 21 & 4 & 0 & 3,7 & 3,8 & 3,9 \\
\hline 38 & 4 & 11 & 2 & 2 & 2 & 20 & 44 & 6 & 4 & 2 & 3,6 & 3,5 & 3,7 \\
\hline 39 & 4 & 12 & 5 & 0 & 0 & 20 & 48 & 15 & 0 & 0 & 4,0 & 3,6 & 3,9 \\
\hline 40 & 1 & 3 & 10 & 4 & 3 & 1 & 6 & 30 & 16 & 15 & 3,2 & 3,2 & 3,3 \\
\hline 41 & 4 & 14 & 2 & 0 & 1 & 20 & 56 & 6 & 0 & 1 & 4,0 & 4,0 & 4,1 \\
\hline 42 & 2 & 4 & 10 & 3 & 2 & 10 & 16 & 30 & 6 & 2 & 3,0 & 3,2 & 3,1 \\
\hline 43 & 3 & 14 & 2 & 1 & 1 & 15 & 56 & 6 & 2 & 1 & 3,8 & 3,9 & 4,2 \\
\hline 44 & 5 & 10 & 4 & 2 & 0 & 25 & 40 & 12 & 4 & 0 & 3,9 & 4,1 & 44 \\
\hline 45 & 12 & 8 & 1 & 0 & 0 & 60 & 32 & 3 & 0 & 0 & 4,5 & 4,0 & 45 \\
\hline
\end{tabular}

En los ítems 6, 7 y 20,por estar construidos de forma negativa, los cálculos se hacen a la inversa, para obtener siempre una escala positiva. En el caso del ítem 6 (la ciencia es aburrida), el cambio convierte la afirmación en la ciencia es entretenida o, en su defecto, la ciencia $\mathrm{NO}$ es aburrida, y un promedio de 4,3 sugiere una actitud muy positiva. Siguiendo un procedimiento similar, se trabaja con el resultado de todos los grados, tanto para hombres como para mujeres, para obtener el promedio general por ítem para toda la muestra. Antes de discutir los promedios generales, se evalúa la consistencia de la prueba con la muestra de estudiantes de la tabla 2. En el anexo 2 se presentan los resultados de cada 
estudiante para cada ítem según la escala de Likert establecida. En esta medida, por ejemplo, el estudiante 1 estuvo de acuerdo (A) con el ítem 1, por lo que aparece un valor de 4, en el ítem 2 estuvo indeciso (I), y por ello aparece 3 , y así sucesivamente para el resto de ítems. En la columna vertical VAR del anexo 2 aparece la varianza de todas las respuestas de los 21 estudiantes para el ítem 1; en la última fila, el valor SUMA corresponde a la suma de todos los valores según la escala para cada estudiante. Obteniendo la varianza de cada ítem y con la suma de las varianzas, se emplea la siguiente ecuación para calcular el parámetro alfa de Cronbach $(\alpha)$, usado para estimar la consistencia interna de la prueba aplicada:

$$
\alpha=\left[\frac{K}{K-1}\right] \times\left|1-\frac{\sum V_{i}}{V_{t}}\right| \text { (valor absoluto) }
$$

$K$ es el número de ítems, $\Sigma V_{i}$ es la sumatoria de la varianza de los ítems; $V_{f}$ es la varianza total delos ítems.

$$
\alpha=\left[\frac{45}{45-1}\right] \times\left|1-\frac{37,8}{156}\right|=0,77
$$

El valor de alfa, igual a 0,77 para esta muestra, indica una consistencia interna adecuada para este estudio (Rodríguez, Jiménez y Caicedo, 2007). En este sentido, Cervantes (2005) está de acuerdo con que un alfa mayor a 0,7 es aceptado en un test psicométrico para hacer válida una prueba. El alfa de Cronbach para la población es de 0,83 , indicando más de un $80 \%$ de consistencia entre los ítems, el cual, comparado con el obtenido por Kind, Jones y Barmby (2007) de 0,93 (para el interés combinado por la ciencia, con tres categorías: aprendizaje, ciencia e importancia) es bueno, si consideramos que en el cálculo para el presente trabajo se introdujo una categoría adicional: el sentido de pertenencia hacia la institución, el cual puede llegar a ser muy subjetivo. Los valores de alfa por categoría y su comparación con la aplicación hecha por Barmby, Kind y Jones (2008) aparecen en la tabla 3. En general, todos los valores son aceptables y nos muestran que, adicional al juicio de expertos, el cuestionario es confiable para ser aplicado.

Tabla 3. Consistencia interna del cuestionario

\begin{tabular}{lcc}
\multicolumn{1}{c}{ Categoría de actitud } & $\begin{array}{c}\text { Alfa de Cronbach en } \\
\text { el presente estudio }\end{array}$ & $\begin{array}{c}\text { Alfa comparativo } \\
\text { Barmby, Kind y Jones (2008) }\end{array}$ \\
\hline Aprendizaje de la ciencia en la escuela. & 0,83 & 0,89 \\
\hline Trabajo práctico en ciencia. & 0,88 & 0,85 \\
\hline Ciencia fuera de la escuela. & 0,79 & 0,88 \\
\hline Importancia de la ciencia. & 0,81 & 0,77 \\
\hline Autoconcepto de ciencia. & 0,76 & 0,85 \\
\hline Futura participación en ciencia. & 0,85 & 0,86 \\
\hline
\end{tabular}

En la tabla 2 aparecen los promedios generales de cada ítem por género. En adelante se empleará $\mathrm{H}$ para indicar los resultados correspondientes al género masculino (hombres) y $M$ para indicar los resultados del género femenino (mujeres). Con base en estos resultados, se evidencia que las diferencias de puntuación por género son pequeñas en contraposición con lo encontrado por otros autores (Vázquez y Manassero, 2008 y George, 2006), quienes otorgan mayor énfasis a las diferencias entre hombres y mujeres. Al respecto, estudios como los de Mason y Kahle (1988) muestran que el sesgo entre géneros desaparece y resaltan que las actitudes hacia la ciencia están más influenciadas por variables como el 
profesorado. Por otra parte, sobre los valores promedio, se observa que el ítem 32 (la ciencia y la tecnología son importantes para la sociedad) posee el mayor valor $(4,7 \mathrm{H}$ y $4,7 \mathrm{M})$, lo cual no es sorprendente si este resultado se relaciona con otros trabajos en los que se descubre que en la escuela sele da un papel preponderante a la ciencia y a la tecnología (Aguirre, 2005). Sin embargo, aunque la ciencia y la tecnología se consideran importantes, el instrumento muestra que no son vistas como un factor que socialmente afecte a toda la población (ítem 35: la ciencia y la tecnología ayudan a aliviar la pobreza, con valores de $3,1 \mathrm{H} \mathrm{y}$ $3,2 \mathrm{M})$. Por otro lado, el ítem 30 (me agradaría volverme un profesor de ciencias) está dentro de los promedios más bajos (con valores de $2,6 \mathrm{H}$ y $3,0 \mathrm{M}$ ), mostrando la escasa inclinación por la actividad de ser docente de ciencias. Sin embargo, este resultado no se puede interpretar como una actitud desfavorable hacia el profesor, pero sí por lo que enseña, por aquella labor que para los estudiantes representa una dificultad (ítem 7, la ciencia me parece difícil).

En la tabla 4, se presentan los valores promedio para las actitudes en las diferentes categorías por género, y en la figura 1 se representa el promedio de los dos géneros en función del grado escolar. De forma general, se puede afirmar que las actitudes decaen entre grado quinto y grado undécimo, lo cual concuerda con lo encontrado por otros autores (Vázquez y Manassero, 2008, Kind, Jones y Barmby, 2007).

Tabla 4. Valores promedio para las categorías de actitud en cada grado

\begin{tabular}{|c|c|c|c|c|c|c|c|c|}
\hline Mujeres & Quinto & Sexto & Séptimo & Octavo & Noveno & Décimo & Undécimo & $\begin{array}{c}\text { Promedio total } \\
\text { grados quinto a } \\
\text { undécimo }\end{array}$ \\
\hline Aprendizaje de la ciencia en la escuela. & 4,2 & 4,0 & 4,0 & 3,7 & 3,9 & 3,9 & 3,7 & 3,9 \\
\hline Autoconcepto de ciencia. & 3,9 & 3,5 & 3,7 & 3,8 & 3,8 & 3,6 & 3,6 & 3,7 \\
\hline Trabajo práctico. & 4,4 & 4,3 & 4,3 & 4,1 & 4,3 & 4,0 & 4,0 & 4,2 \\
\hline Ciencia fuera de la escuela. & 4,1 & 4,0 & 3,7 & 3,6 & 3,7 & 3,7 & 3,9 & 3,8 \\
\hline Futura participación en ciencia. & 3,6 & 3,5 & 2,9 & 3,1 & 3,1 & 3,0 & 3,2 & 3,2 \\
\hline Importancia de la ciencia. & 4,0 & 4,0 & 3,7 & 3,8 & 3,9 & 4,0 & 3,8 & 3,9 \\
\hline Pertenencia a la institución. & 4,0 & 3,6 & 3,6 & 3,5 & 3,7 & 3,5 & 3,7 & 3,6 \\
\hline Interés combinado. & 4,0 & 3,8 & 3,6 & 3,5 & 3,6 & 3,5 & 3,6 & 3,7 \\
\hline Hombres. & $5^{\circ}$ & $6^{\circ}$ & $7^{\circ}$ & $8^{\circ}$ & $9^{\circ}$ & $10^{\circ}$ & $11^{\circ}$ & Promedio total \\
\hline Aprendizaje de la ciencia en la escuela. & 4,1 & 4,0 & 4,0 & 3,8 & 3,9 & 3,8 & 3,2 & 3,8 \\
\hline Autoconcepto de ciencia. & 3,8 & 3,7 & 3,9 & 3,7 & 3,9 & 3,6 & 3,2 & 3,7 \\
\hline Trabajo práctico. & 4,3 & 4,3 & 4,3 & 4,2 & 4,4 & 4,0 & 3,6 & 4,1 \\
\hline Ciencia fuera de la escuela. & 3,8 & 3,9 & 3,6 & 3,6 & 3,6 & 3,7 & 3,4 & 3,7 \\
\hline Futura participación en ciencia. & 3,6 & 3,3 & 2,7 & 3,1 & 3,1 & 3,0 & 2,8 & 3,1 \\
\hline Importancia de la ciencia. & 4,0 & 3,9 & 3,6 & 3,8 & 3,9 & 4,0 & 3,8 & 3,8 \\
\hline Pertenencia a la institución. & 3,8 & 3,7 & 3,7 & 3,5 & 3,7 & 3,4 & 3,4 & 3,6 \\
\hline Interés combinado. & 3,8 & 3,7 & 3,4 & 3,5 & 3,6 & 3,5 & 3,1 & 3,5 \\
\hline
\end{tabular}


La figura 1 muestra que la categoría de actitud que más decae es la futura participación en ciencia, que inicia con un valor positivo en grado quinto $(3,6$ en general) y ya en grado séptimo decae a la zona negativa $(2,8$ en promedio, tabla 4), tanto para hombres como para mujeres, y se mantiene su tendencia hasta grado undécimo, conduciendo a valores de $3,1 \mathrm{H}$ y $3,2 \mathrm{M}$ como promedios totales del ciclo de estudios. Al realizarse la entrevista grupal, se logró confrontar a los estudiantes sobre sus perspectivas en ciencias y casi por unanimidad todos manifestaron no tener planes de estudiar alguna carrera científica; por ejemplo, química. Consideran poco interesante la profesión de científico, opinan que no debe ser asumida por ellos, aunque a juicio del grupo focal la ciencia sea espectacular, sobre todo al referirse a lo que ven en los canales televisivos de divulgación. Muestran su convicción de dejar esa actividad a otras personas mejor preparadas y con capacidades sobresalientes. Durante la entrevista, también manifestaron que, si bien la ciencia soluciona problemas, por el hecho de considerarla difícil de aprender no piensan dedicarle tiempo y esfuerzo a formarse en ella, en vista de que la consideran muy teórica. Al respecto, un estudiante afirma:"si en el colegio es tan difícil, en la universidad será más difícil; es solo para los inteligentes". La idea de dificultad como característica importante de la ciencia es encontrada por Molina, Carriazo y Farias (2011) en un estudio realizado con estudiantes de diferentes profesiones en su primer curso de química general, revelando que la dificultad en el estudio de la química es la categoría que más aleja a los estudiantes de una actitud favorable frente a los cursos de química o de la posibilidad de seguir tomando cursos de química fuera de los estrictamente obligatorios.

Figura 1. Variación de las actitudes hacia la ciencia según el año escolar

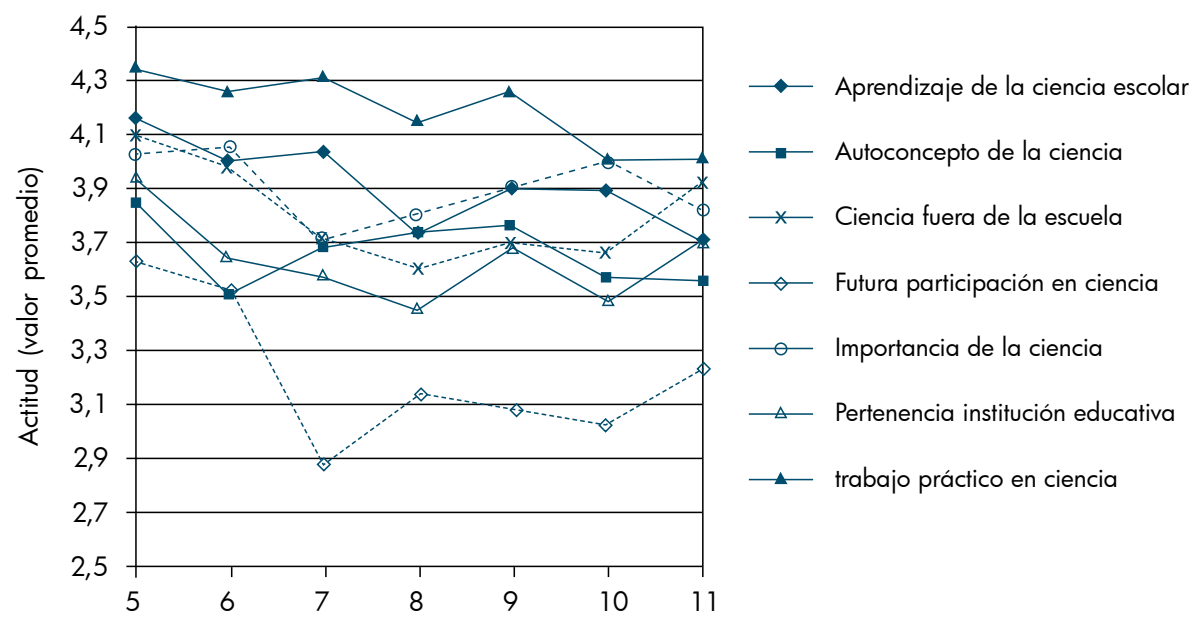

Con respecto a este resultado, que indica baja actitud frente a las carreras científicas (ítem 31: me gustaría ser un científico, con valores de $3,0 \mathrm{H}$ y $3,1 \mathrm{M}$ ), se encuentra similitud con resultados como los de Barmby, Kind y Jones (2008), reflejando posiblemente una tendencia mundial que no está influenciada por cuestiones económicas, aunque el estu- dio ROSE (Schreiner y Sjøberg, 2004) mostró que dicha tendencia es mayor en países más industrializados:

Los jóvenes de los países desarrollados piensan que las ciencias son importantes, pero la gran mayoría no quiere continuar estudiándolas más allá de la 
etapa obligatoria, mientras que en los países en vía de desarrollo aún hay un amplio sector que piensa seguir estudiándolas (Marbá y Márquez, 2010).

Con base en dichas argumentaciones, se puede ratificar que, en términos generales, la mayoría de los estudiantes no tiene entre sus planes las carreras relacionadas con la ciencia o con trabajos que impliquen ciencia y tecnología.

En contraste con otros autores, como se mencionó antes, los resultados de género no marcan una mayor relevancia en cuanto a los valores promedio de la futura participación en ciencia, mostrándose contrario a aquellos estudios que indican claramente una actitud más positiva para los hombres respecto a las mujeres (Vázquez y Manassero, 2008 y George, 2006). En buena medida, esto indica que, en la población de estudio, el género no es la variable que ejerce influencia considerable en las actitudes y que las diferencias encontradas están relacionadas con otros factores.

La mejor actitud hacia la ciencia se encuentra en el trabajo práctico, siendo casi constante de grado quinto a grado noveno, con valores muy positivos, pero decae un poco entre los grados décimo y undécimo. En la entrevista grupal, se mostró la importancia del componente práctico en temas relativos a la ciencia; los estudiantes manifestaron su valoración hacia las actividades prácticas, ya que motivan y le otorgan una dimensión atractiva a la ciencia al pasar de fórmulas, nombres y cálculos tediosos y abstractos a cuestiones concretas, que no obligan a memorizar sin sentido. Todos indicaron que si las clases tuviesen más contribución práctica serían más atractivas (ítem 17: me gustaría tener más trabajo experimental en clases de ciencias, con un valor general de 4,3 ). Sobre esto, se recomienda convertir el aula de ciencias en un espacio que utilice más la actividad práctica dirigida por el profesor, para incentivar la curiosidad y la asociación de conceptos, similar a las actividades prácticas utilizadas en el grupo focal, con el cual se trabajó en la generación de oxígeno a partir de levadura y peróxido de hidrogeno, para discutir el papel de este gas en la atmósfera y en la vida. Los estudiantes mostraron cierta inquietud porque, según ellos, son poco frecuentes las sesiones prácticas recibidas, tal como lo revelaron Molina y Farías (2005), al encontrar que en los grados décimo y undécimo de gran número de instituciones en Bogotá (Colombia) solo se realizan de dos a tres trabajos prácticos en cada curso durante el año.

En el trabajo práctico, el ítem 18 (el trabajo experimental me facilita el aprendizaje de las ciencias) obtiene los mejores promedios $(4,4 \mathrm{M}$ y $4,5 \mathrm{H})$, corroborando lo encontrado en las entrevistas y en otros estudios (Reid y Skyabina, 2002) donde la preferencia por los trabajos prácticos se da como forma de explicar los fenómenos, en contraste a solo utilizar palabras.

La ciencia escolar ha sido un aspecto relevante en varios estudios (Kind, Jones y Barmby, 2007; Bennett, 2003) debido a su relación directa con lo ocurrido en el aula, que no necesariamente corresponde al concepto personal que se tenga de ciencia. Los resultados muestran que la ciencia escolar (aprendizaje de la ciencia 
en la escuela) inicia con una actitud positiva (4,2 en grado quinto), pero decae sucesivamente hasta el final de los estudios, mostrando valores de $3,7 \mathrm{M}$ y $3,2 \mathrm{H}$ en grado undécimo. Aunque los resultados no muestran diferencias importantes entre géneros, en grado undécimo las mujeres terminan con una actitud un poco más positiva hacia el aprendizaje de la ciencia. Los ítems 1 y 3 de la categoría aprendizaje de la ciencia en la escuela están a la cabeza de las actitudes positivas $(4,6$ y 4,4 en promedio, tabla 2) con valores cercanos entre géneros. Entre grados quinto y séptimo, las actitudes se mantienen, pero en grado octavo decaen, esto relacionado quizá con los cambios propios de la adolescencia (el grado octavo presenta los valores mínimos para casi todas las categorías, excepto para la categoría futura participación en ciencias). En la tabla 4, se observa que el género no afecta los promedios de forma importante, y en algunos casos las mujeres poseen valores más altos.

El auto-concepto de ciencia se mantiene casi estable durante los años escolares, cayendo solo un poco para los hombres en grado undécimo a un valor casi neutro de actitud (3,2).El ítem 9 (obtengo buenas notas en ciencias) da los valores más altos en esta categoría $(4,0)$, lo cual puede parecer contradictorio si pensamos en una relación entre dificultad y desempeño, ya que el ítem 7 (la ciencia me parece difícil) marca valores bajos (3,5 en promedio) y el 11 (la ciencia es mi tema favorito), de forma similar $(3,3)$. Los valores por sí solos no pueden explicar totalmente la posible contradicción entre los resultados académicos y la actitud, y solo en la entrevista es posible saber que los resultados en las notas no son necesariamente un buen reflejo de los desempeños, ya que en general las calificaciones dependen de actividades como trabajos escritos, informes, etc., que generan calificaciones más altas que las pruebas escritas directas sobre contenidos científicos. Con respecto a estos resultados, se abre una nueva posibilidad de estudio, en la que se contemplen profundamente el diseño del currículo y su relación con las actitudes de los estudiantes y, sobre todo, la influencia de dichas actitudes en el desarrollo de las intencionalidades curriculares en el aula.

La ciencia fuera de la escuela se mantiene con actitudes positivas durante la secundaria, aunque decae desde grado quinto a grado décimo, y sube en grado undécimo levemente. Esta categoría la conforman los ítems 21 a 26, en los que 23 y 26 marcan los mayores valores. En los resultados sobre el ítem 23 (me gustaría visitar museos científicos, con valores de 4,2H y $4,5 \mathrm{M})$, se comprende perfectamente cuando los estudiantes manifiestan querer una ciencia más activa, más relacionada con su entorno, donde se pueda interactuar con materiales cotidianos y se ofrezcan datos curiosos. De esta manera, este tipo de visitas mejora la actitud hacia las ciencias y puede potenciar enormemente el aprendizaje, ya que genera un espacio para la comunicación y mejora la adquisición de contenidos (Varela y Stegler, 2004).

Sobre el ítem 26 (es emocionante e interesante aprender sobre los nuevos avances y descubrimientos en ciencias, con valores de $4,2 \mathrm{H}$ y $4,3 \mathrm{M})$, es cuestionable que el deseo de aprender nuevos hechos sobre ciencia no esté de acuerdo con adelantar actividades al respecto, como leer o ver TV (ítems 22 y 25), por lo que se puede inferir que existe una actitud pasiva de los estudiantes, quienes probablemente esperan satisfacer el deseo de aprender solo mediante la intervención directa del profesor en la clase de ciencias. De este modo, se requiere involucrar más en las clases de ciencias la información científica presentada en los medios de comunicación masiva, para posibilitar una mejor alfabetización científica (Walczak, 2007) e incorporar 
escenarios alternos para el desarrollo de competencias, tales como la digital, la social y la ciudadana.

La comparación entre la ciencia escolar y la ciencia fuera de la escuela deja ver que esta última no se asume como altamente importante, lo que puede indicar poco interés en temas que superen los aspectos obligatorios sobre el aprendizaje de las ciencias. Entonces, es necesario estimular la implementación de actividades extraescolares (ítem 24: me gustaría realizar más actividades científicas fuera de la clase, con valores de $4,0 \mathrm{H}$ y $4,1 \mathrm{M}$ ) en las que la escuela, la casa y los medios puedan involucrase y relacionarse, dándose así una mayor interacción entre la ciencia y el medio social.

En cuanto a la encuesta, las preguntas de pertenencia escolar dentro del cuestionario buscaban ir más allá de las clases de ciencias, para contar con información que relacionara las actitudes con el contexto escolar. Aunque la actitud de pertenencia decae de quinto a octavo, esta sube un poco en grado undécimo (figura 1), en relación quizá con un mayor criterio para valorar la institución. En este mismo plano de la discusión, el ítem 42 (del total de mi tiempo, deseo permanecer mucho en el colegio) muestra al colegio como un lugar poco atractivo o poco acogedor, relacionado más con el desarrollo del currículo escolar (ítem 40) que con el ambiente social (ítems 38 y 44).

Para consolidar el análisis, se integraron tres categorías de actitud (ciencia escolar, ciencia fuera de la escuela y futura participación) para obtener el denominado interés combinado hacia la ciencia. En la figura 2, se representa ese interés según el grado escolar para ambos géneros, sin buscar diferencias entre estos, sino tratando de establecer tendencias para conocer el desarrollo de las actitudes generales. Como se indicó antes, las actitudes (interés combinado hacia las ciencias) decaen progresivamente hasta grado octavo y luego aumentan levemente hasta grado undécimo, partiendo inicialmente de una actitud positiva y culminando con una actitud moderadamente positiva.

Figura 2. Interés combinando hacia la ciencia (ICC) según el grado escolar

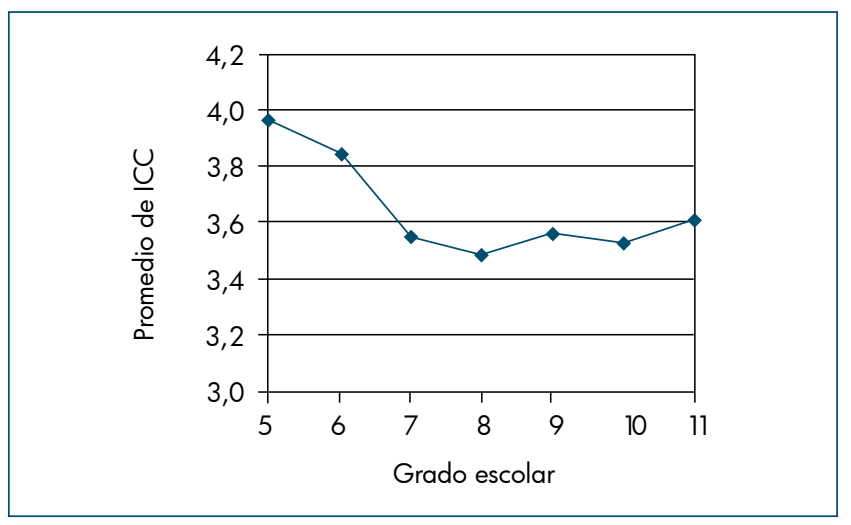

La disminución de actitudes favorables hacia la ciencia constituye un enemigo secreto en la enseñanza de las ciencias, que puede convertirse en un problema 
mayor en la medida en que se ignore el pensamiento y las creencias de los estudiantes sobre el quehacer científico.

\section{Conclusiones}

Se adaptó y aplicó un instrumento para evaluar las actitudes hacia la ciencia en estudiantes de grado quinto a undécimo (educación secundaria colombiana), con un coeficiente de confiabilidad (alfa de Cronbach, $\alpha=0,77$ ) que garantizó una adecuada consistencia interna en este (el valor mínimo estadísticamente aceptado es de 0,70). En términos generales, se encontró una disminución progresiva importante de las actitudes favorables hacia la ciencia desde grado quinto a undécimo. Con respecto a la evolución de concepciones sobre ciencia, se puede concluir que hay un descenso progresivo desde quinto hasta undécimo, lo que concuerda con resultados obtenidos en estudios similares
(Vázquez y Manassero, 2008; Kind, Jones y Barmby, 2007). Por otra parte, no se encontraron marcadas diferencias en las concepciones sobre ciencia en hombres y mujeres, además de evidenciarse que se le otorga gran importancia a la ciencia y a la tecnología, pero poca valoración al interés futuro de trabajar en ellas, coherente con la percepción de dificultad en estos campos. Lo anterior se complementa con la recurrencia en el sentimiento manifestado por el colectivo de estudiantes del grupo focal (entrevistados), consistente en ver la ciencia como un campo de trabajo complejo y poco atractivo. Finalmente, se encontró una actitud favorable hacia la actividad práctica en ciencias, mayor en los primeros cinco grados (quinto a noveno) que en los dos últimos (décimo y undécimo) y fuertemente influenciada por la percepción del escaso trabajo experimental durante toda la etapa escolar y el deseo de recibir un volumen mayor de actividades prácticas.

\section{Referencias Bibliográficas}

Aguirre, J. (2005). La percepción que tienen los colombianos sobre la ciencia y la tecnología. Colciencias. Bogotá: Panamericana.

Barmby, P., Kind, P. y Jones, K. (2008). Examining changing attitudes in secondary school science. International Journal of Science Education, 30 (8), 1075-1093.

Bennett, J. (2003). Teaching and learning Science. Londres: Continuum.

Cervantes, V. (2005). Interpretaciones del coeficiente alfa de Cronbach. Avances en Medición, 3, 9-28.

Cheung, D. (2009). Students' attitudes toward chemistry lessons: the interaction effect between grade level and gender. Research in Science Education, 39, 75-91.

Cukrowska, E., Staskun, M. y Schoeman, H. (1999). Attitudes towards chemistry and their relationship to student achievement in introductory chemistry courses. South African Journal of Chemistry, 52 (1),8-15.

Fensham, P. (2004). Beyond knowledge: other scientific qualities as outcomes for school science education. En R.M. Janiuk y E. Samonek-Miciuk (Eds.). Science and technology education for a diverse world-dilemmas, needs and partnerships. International Organization for Science and Technology Education (IOSTE) Xlth Symposium Proceedings (pp. 23-25). Lublin, Polonia: Marie Curie-Sklodowska University Press.

Freedman, M. (1997). Relationship among laboratory instruction, attitude toward science, and achievement in science knowledge. Journal of Research in Science Teaching, 34 (4), 343-357. 
George, R. (2006). A cross domain analysis of change in students' attitudes toward science and attitudes about the utility of science. International Journal of Science Education, 28 (6), 571-589.

Hambleton, R. (2002). Adapting achievement tests into multiple languages for international assessments. En A.C. Porter, y A. Gamoran (Eds.). Methodological advances in cross-national surveys of educational achievement (p. 58). Washington: National Academy of Sciences.

Hofstein, A. y Mamlok R. (201 1). High-school students' attitudes toward and interest in learning chemistry. Educación Química, 22, 90-102.

Kaya, O., Yager, R. y Dogan, A. (2009). Changes in attitudes towards science-technology-society of pre-service science teachers. Research in Science Education, 39, 257-279.

Kidman, G. (2009). Attitudes and interests toward biotechnology: the mismatch between students and teachers. Eurasia Journal of Mathematics, Science and Technology Education, 5, 135-143.

Kind, P., Jones, K. y Barmby, P. (2007). Developing attitudes towards science Measurements. International Journal of Science Education, 29 (7), 871-893.

Kurbanoglu, N. y Akim, A. (2010). The relationships between university students' chemistry laboratory anxiety, attitudes, and self-efficacy beliefs. Australian Journal of Teacher Education, 35, 48-59.

Marbà-Tallada, A. y Márquez-Bargalló, C. (2010). ¿̇Qué opinan los estudiantes de las clases de ciencias? Un estudio transversal de sexto de primaria a cuarto de ESO. Enseñanza de las Ciencias, 28 (1), 19-30

Mason, C. y Kahle, J. (1988). Student attitudes toward science and science-related careers: a program designed to promote a stimulating gender-free learning enviroment. Journal of Research in Science Teaching, 26 (1), 25-39.

Mirandes, J.; Sanmartí, N. y Tarín, R. (1993). Diferents expectatives del professorat i del propi alumnat sobre el seu futur profesional. Enseñanza de las Ciencias, número extraordianario, 103-104.

Molina, M. y Farias, D. (2005). Conocimiento de la importancia del trabajo experimental en la enseñanza de la química en la educación secundaria. Tecne, Episteme y Didaxis, número extraordinario, 145.

Molina, M.; Carriazo, J. y Farias, D. (2011). Actitudes hacia la química de estudiantes de diferentes carreras universitarias en Colombia. En prensa. Química Nova.

Murphy, C. y Beggs, J. (2006). Children perceptions of school science. School Science Review, 84 (308), 109-116.

Pell, T. y Jarvis, T. (2001). Developing attitude to science scales for use with children of ages from five to eleven years. International Journal of Science Education, 23, 847- 862 
Prieto, L. y Vera, L. (2008). Actitudes hacia la ciencia en estudiantes de secundaria. Psychologia: Avances en la Disciplina, 2 (1), 133-160.

Reid, N. (2006). Thoughts on attitude measurement. Research in Science \& Technological Education, $24(1), 3-27$.

Reid, N. y Skryabina, E. (2002). Attitudes towards physics. Research in Science \& Tecnological Education, $20(1), 68-81$.

Rodríguez, W., Jiménez, R. y Caicedo, C. (2007). Protocolo de actitudes relacionadas con la ciencia: adaptación para Colombia. Psychologia: Avances en la Disciplina, 1 (2), 85-100.

Salta, K. y Tzougraki, C. (2004). Attitudes toward chemistry among 11 th grade students in high schools in Greece. Science Education, 88, 535-547.

Schreiner, C. y Sjøberg, S. (2004). Sowing the seeds of ROSE. Background, Rationale, Questionaire Development and Data Collection for ROSE (The Relevance of Science Education) - A comparative study of students' views of science and science education. Acta Didactica. (4/2004), Dept. of Teacher Education and School Development, University of Oslo, Norway. Recuperado de:http:// www.ils.uio.no/forskning/rose/documents/AD0404.pdf

Tekbiyik, A. y Akdeniz, A. (2010). A contemporary physics attitude scale for secondary school students: development, validity and reliability. Journal of Turkish Science Education, 7, 145-147.

Varela, C. y Stengler, E. (2004). Los museos interactivos como recurso didáctico: El Museo de las Ciencias y el Cosmos. Revista Eureka Enseñanza y Divulgación de las Ciencias, 3 (1), 32-47.

Vázquez, A. y Manassero, M. (2008). El declive de las actitudes hacia la ciencia de los estudiantes: un indicador inquietante para la educación científica. Revista Eureka Enseñanza y Divulgación de las Ciencias, 5 (3), 274-292.

Walczak, M. (2007). Using news assignments to develop skills for learning about science from public sources. Journal of Chemical Education, 84 (6), 961-966.

Wang, T. y Berlin, D. (2010). Construction and validation of an instrument to measure Taiwanese elementary students' attitudes toward their science class. International Journal of Science Education, 32 (18), 2413-2428. 


\section{Anexo 1. Cuestionario de actitudes relacionadas con la ciencia.}

Este cuestionario contiene enunciados y afirmaciones acerca de las actitudes en ciencias. Deseamos saber tu opinión personal en cada uno de los casos, para los cuales no existe una respuesta correcta o única, solo lo que piensas y sientes. Lee atentamente cada enunciado y señala con una $X$, en el cuadro respectivo, tu respuesta.Posees las siguientes opciones:

$\mathrm{TA}=$ totalmente de acuerdo. $\mathrm{A}=$ de acuerdo. $I=$ no estoy seguro $(\mathrm{a})$, indecisión.

$\mathrm{D}=$ en desacuerdo. $\mathrm{TD}=$ totalmente en desacuerdo.

Edad: Genero: Masculino Femenino Institución educativa:

Grado:

\begin{tabular}{|c|c|c|c|c|c|c|}
\hline 1 & En las clases de ciencias aprendemos cosas interesantes. & TA & A & I & D & TD \\
\hline 2 & En casa, reviso mis apuntes tomados en las clases de ciencias. & TA & A & I & D & TD \\
\hline 3 & Las clases de ciencias son interesantes. & TA & A & 1 & D & TD \\
\hline 4 & Me gustaría tener más clases de ciencias en la semana. & TA & A & 1 & D & TD \\
\hline 5 & Me agrada más la clase de cienciasque otras asignaturas o materias. & TA & A & 1 & D & TD \\
\hline 6 & La ciencia es aburrida. & TA & A & I & D & TD \\
\hline 7 & La ciencia me parece difícil. & TA & A & 1 & D & TD \\
\hline 8 & Me creo bueno (a) en ciencias. & TA & A & I & D & TD \\
\hline 9 & Obtengo buenas notas en ciencias. & TA & A & I & D & TD \\
\hline 10 & Aprendo ciencias con rapidez. & TA & A & I & D & TD \\
\hline 11 & La ciencia es mi tema favorito. & TA & A & I & D & TD \\
\hline 12 & En mis clases de ciencias, comprendo todos los contenidos. & TA & A & I & D & TD \\
\hline 13 & El trabajo experimental en ciencias es emocionante. & TA & A & I & D & TD \\
\hline 14 & $\begin{array}{l}\text { Me gusta el trabajo experimental en ciencias porque me genera expectativas y } \\
\text { preguntas. }\end{array}$ & TA & A & I & D & TD \\
\hline 15 & $\begin{array}{l}\text { El trabajo experimental en ciencias es agradable porque me permite trabajar en } \\
\text { grupo. }\end{array}$ & TA & A & I & D & TD \\
\hline 16 & $\begin{array}{l}\text { Cuando realizamos trabajo experimental en ciencias, me agrada porque puedo } \\
\text { planearlo. }\end{array}$ & TA & A & I & D & TD \\
\hline 17 & Me gustaría tener más trabajo experimental en clases de ciencias. & TA & A & । & $\mathrm{D}$ & TD \\
\hline 18 & El trabajo experimental me facilita el aprendizaje de las ciencias. & TA & A & I & D & TD \\
\hline 19 & Espero con interés las siguientes actividades experimentales en ciencias. & TA & A & I & D & TD \\
\hline 20 & El trabajo experimental en ciencias es aburrido. & TA & A & I & D & TD \\
\hline 21 & Me gustaría pertenecer a un club de ciencias. & TA & A & I & D & TD \\
\hline 22 & Me agrada ver programas de ciencias en la TV. & TA & A & I & D & TD \\
\hline 23 & Me gustaría visitar museos científicos. & TA & A & । & D & TD \\
\hline
\end{tabular}




\begin{tabular}{|c|c|c|c|c|c|c|}
\hline 24 & Me gustaría realizar más actividades científicas fuera de la clase. & TA & A & 1 & $\mathrm{D}$ & TD \\
\hline 25 & Me gustaría leer libros y revistas de ciencias o de divulgación científica. & TA & A & 1 & $\mathrm{D}$ & TD \\
\hline 26 & $\begin{array}{l}\text { Es emocionante e interesante aprender sobre los nuevos avances y } \\
\text { descubrimientos en ciencias. }\end{array}$ & TA & A & I & $\mathrm{D}$ & TD \\
\hline 27 & Me gustaría estudiar más ciencias en el futuro. & TA & A & I & $\mathrm{D}$ & TD \\
\hline 28 & Me agradaría estudiar una carrera científica en la universidad. & TA & A & 1 & $\mathrm{D}$ & TD \\
\hline 29 & Me gustaría tener un empleo relacionado con las ciencias. & TA & A & 1 & $\mathrm{D}$ & TD \\
\hline 30 & Me agradaría volverme un profesor de ciencias. & TA & A & I & $\mathrm{D}$ & TD \\
\hline 31 & Me gustaría ser un científico. & TA & A & 1 & $\mathrm{D}$ & TD \\
\hline 32 & La ciencia y la tecnología son importantes para la sociedad. & TA & A & 1 & $\mathrm{D}$ & TD \\
\hline 33 & La ciencia y la tecnología hacen la vida más fácil y confortable. & TA & A & I & $\mathrm{D}$ & TD \\
\hline 34 & Los beneficios de las ciencias son más importantes que los efectos perjudiciales. & TA & A & 1 & $\mathrm{D}$ & TD \\
\hline 35 & La ciencia y la tecnología ayudan a aliviar la pobreza. & TA & A & I & $\mathrm{D}$ & TD \\
\hline 36 & Hay muchas cosas importantes que ocurren en ciencia y tecnología. & TA & A & 1 & $\mathrm{D}$ & TD \\
\hline 37 & El trabajo de los científicos es emocionante. & TA & A & I & $\mathrm{D}$ & TD \\
\hline 38 & Me agrada el ambiente escolar de mi colegio. & TA & A & 1 & $\mathrm{D}$ & TD \\
\hline 39 & Recomendaría a mis amigos estudiar en este colegio. & TA & A & 1 & $\mathrm{D}$ & TD \\
\hline 40 & Las actividades que realizamos en el colegio me parecen aburridas. & TA & A & 1 & $\mathrm{D}$ & TD \\
\hline 41 & Me siento parte de esta institución educativa. & TA & A & I & $\mathrm{D}$ & TD \\
\hline 42 & Del total de mi tiempo, deseo permanecer mucho en el colegio. & TA & A & 1 & $\mathrm{D}$ & TD \\
\hline 43 & Me llevo bien con mis profesores. & TA & A & I & $\mathrm{D}$ & TD \\
\hline 44 & Me siento feliz la mayor parte del tiempo cuando estoy en el colegio. & TA & A & I & $\mathrm{D}$ & TD \\
\hline 45 & Doy todo lo necesario para tener buen rendimiento en el colegio. & TA & A & I & $\mathrm{D}$ & TD \\
\hline
\end{tabular}




\section{Anexo 2. Valoración de lasrespuestas de los estudiantes según la escala de Likert y la varianza*.}

\begin{tabular}{|c|c|c|c|c|c|c|c|c|c|c|c|c|c|c|c|c|c|c|c|c|c|c|}
\hline Est/it & 1 & 2 & 3 & 4 & 5 & 6 & 7 & 8 & 9 & 10 & 11 & 12 & 13 & 14 & 15 & 16 & 17 & 18 & 19 & 20 & 21 & VAR \\
\hline 1 & 4 & 4 & 4 & 5 & 5 & 4 & 4 & 4 & 5 & 5 & 5 & 5 & 5 & 5 & 5 & 5 & 5 & 4 & 5 & 4 & 5 & 0,25 \\
\hline 2 & 3 & 3 & 2 & 4 & 4 & 4 & 2 & 5 & 4 & 3 & 2 & 2 & 4 & 4 & 3 & 5 & 4 & 3 & 4 & 3 & 4 & 0,86 \\
\hline 3 & 5 & 5 & 4 & 4 & 5 & 4 & 4 & 4 & 4 & 5 & 4 & 5 & 4 & 4 & 5 & 5 & 5 & 4 & 4 & 4 & 4 & 0,25 \\
\hline 4 & 3 & 4 & 5 & 3 & 3 & 5 & 4 & 1 & 4 & 3 & 3 & 3 & 3 & 5 & 2 & 4 & 5 & 3 & 4 & 2 & 5 & 1,26 \\
\hline 5 & 4 & 3 & 4 & 5 & 5 & 4 & 5 & 1 & 3 & 2 & 2 & 3 & 4 & 4 & 1 & 3 & 5 & 2 & 4 & 4 & 5 & 1,66 \\
\hline 6 & 5 & 4 & 5 & 4 & 5 & 4 & 4 & 4 & 4 & 4 & 5 & 5 & 4 & 5 & 5 & 5 & 5 & 4 & 4 & 4 & 2 & 0,53 \\
\hline 7 & 4 & 3 & 3 & 4 & 5 & 5 & 4 & 3 & 4 & 2 & 4 & 4 & 4 & 3 & 3 & 3 & 5 & 4 & 4 & 2 & 2 & 0,86 \\
\hline 8 & 4 & 3 & 4 & 4 & 4 & 5 & 5 & 3 & 4 & 3 & 4 & 4 & 4 & 4 & 4 & 3 & 4 & 3 & 4 & 4 & 4 & 0,33 \\
\hline 9 & 4 & 4 & 4 & 4 & 3 & 4 & 5 & 4 & 4 & 4 & 4 & 5 & 3 & 4 & 5 & 4 & 4 & 3 & 4 & 4 & 4 & 0,30 \\
\hline 10 & 4 & 3 & 4 & 5 & 4 & 4 & 5 & 3 & 5 & 3 & 3 & 4 & 4 & 5 & 5 & 5 & 5 & 3 & 4 & 4 & 3 & 0,65 \\
\hline 11 & 5 & 2 & 4 & 3 & 4 & 5 & 4 & 1 & 3 & 2 & 2 & 3 & 4 & 4 & 2 & 4 & 4 & 1 & 3 & 3 & 4 & 1,36 \\
\hline 12 & 4 & 4 & 5 & 3 & 5 & 4 & 4 & 3 & 3 & 5 & 4 & 4 & 5 & 3 & 5 & 4 & 4 & 3 & 4 & 4 & 4 & 0,50 \\
\hline 13 & 5 & 5 & 5 & 4 & 5 & 5 & 5 & 3 & 5 & 5 & 3 & 5 & 5 & 4 & 5 & 5 & 5 & 4 & 3 & 4 & 5 & 0,56 \\
\hline 14 & 4 & 4 & 4 & 3 & 5 & 4 & 4 & 4 & 5 & 5 & 4 & 4 & 4 & 5 & 5 & 5 & 4 & 4 & 4 & 4 & 4 & 0,29 \\
\hline 15 & 5 & 4 & 4 & 5 & 4 & 4 & 3 & 4 & 5 & 5 & 4 & 5 & 5 & 5 & 5 & 5 & 4 & 4 & 4 & 4 & 5 & 0,36 \\
\hline 16 & 4 & 3 & 5 & 3 & 3 & 4 & 5 & 2 & 4 & 3 & 5 & 2 & 4 & 4 & 4 & 4 & 3 & 3 & 5 & 3 & 5 & 0,91 \\
\hline 17 & 4 & 5 & 5 & 3 & 4 & 5 & 4 & 5 & 4 & 5 & 5 & 3 & 5 & 5 & 5 & 5 & 4 & 4 & 5 & 3 & 5 & 0,56 \\
\hline 18 & 5 & 4 & 5 & 3 & 5 & 5 & 5 & 4 & 5 & 5 & 5 & 4 & 5 & 4 & 1 & 5 & 5 & 4 & 5 & 4 & 5 & 0,96 \\
\hline 19 & 4 & 4 & 5 & 4 & 4 & 5 & 5 & 4 & 4 & 4 & 5 & 4 & 4 & 5 & 5 & 5 & 5 & 3 & 4 & 3 & 5 & 0,43 \\
\hline 20 & 1 & 2 & 1 & 1 & 1 & 1 & 1 & 2 & 1 & 1 & 1 & 2 & 1 & 2 & 1 & 1 & 1 & 2 & 1 & 1 & 1 & 0,19 \\
\hline 21 & 1 & 3 & 2 & 3 & 4 & 4 & 3 & 2 & 5 & 2 & 3 & 3 & 3 & 3 & 3 & 3 & 5 & 1 & 3 & 3 & 2 & 1,09 \\
\hline 22 & 3 & 3 & 4 & 2 & 5 & 5 & 4 & 4 & 5 & 4 & 1 & 5 & 4 & 4 & 2 & 5 & 5 & 2 & 4 & 4 & 4 & 1,39 \\
\hline 23 & 3 & 2 & 5 & 3 & 5 & 5 & 3 & 5 & 5 & 4 & 5 & 3 & 4 & 5 & 4 & 5 & 5 & 4 & 3 & 4 & 5 & 0,93 \\
\hline 24 & 2 & 1 & 1 & 2 & 3 & 1 & 2 & 4 & 2 & 4 & 2 & 3 & 1 & 1 & 4 & 1 & 1 & 4 & 1 & 3 & 2 & 1,33 \\
\hline 25 & 2 & 3 & 2 & 3 & 2 & 2 & 3 & 4 & 1 & 5 & 4 & 4 & 2 & 3 & 1 & 2 & 2 & 4 & 3 & 4 & 5 & 1,39 \\
\hline 26 & 4 & 4 & 4 & 5 & 4 & 4 & 5 & 4 & 5 & 5 & 3 & 3 & 5 & 5 & 5 & 5 & 5 & 3 & 4 & 4 & 4 & 0,51 \\
\hline 27 & 3 & 3 & 5 & 4 & 4 & 5 & 4 & 2 & 4 & 2 & 4 & 2 & 3 & 5 & 2 & 3 & 5 & 3 & 3 & 2 & 4 & 1,16 \\
\hline 28 & 3 & 2 & 4 & 3 & 4 & 5 & 4 & 3 & 5 & 1 & 4 & 3 & 3 & 4 & 2 & 3 & 5 & 2 & 1 & 2 & 5 & 1,59 \\
\hline 29 & 4 & 3 & 4 & 3 & 5 & 4 & 4 & 2 & 5 & 3 & 4 & 3 & 3 & 4 & 5 & 4 & 5 & 2 & 3 & 4 & 5 & 0,89 \\
\hline 30 & 1 & 3 & 2 & 2 & 5 & 1 & 1 & 2 & 4 & 1 & 2 & 2 & 3 & 1 & 2 & 3 & 5 & 2 & 1 & 1 & 2 & 1,56 \\
\hline 31 & 1 & 3 & 4 & 3 & 1 & 5 & 3 & 4 & 4 & 1 & 3 & 2 & 3 & 4 & 4 & 4 & 4 & 2 & 3 & 2 & 4 & 1,35 \\
\hline 32 & 5 & 5 & 5 & 5 & 1 & 5 & 5 & 5 & 5 & 5 & 5 & 4 & 5 & 5 & 5 & 5 & 5 & 4 & 5 & 4 & 5 & 0,83 \\
\hline 33 & 5 & 3 & 5 & 4 & 4 & 5 & 5 & 4 & 5 & 5 & 5 & 3 & 4 & 4 & 5 & 5 & 5 & 4 & 4 & 4 & 5 & 0,46 \\
\hline 34 & 4 & 2 & 4 & 3 & 4 & 4 & 4 & 2 & 4 & 3 & 5 & 2 & 4 & 4 & 4 & 5 & 3 & 3 & 3 & 3 & 4 & 0,76 \\
\hline 35 & 2 & 1 & 2 & 3 & 3 & 3 & 3 & 3 & 1 & 4 & 4 & 2 & 3 & 5 & 3 & 3 & 5 & 3 & 3 & 3 & 4 & 1,10 \\
\hline 36 & 4 & 4 & 5 & 4 & 4 & 5 & 4 & 5 & 5 & 5 & 4 & 3 & 4 & 4 & 5 & 5 & 4 & 4 & 5 & 4 & 4 & 0,33 \\
\hline 37 & 3 & 3 & 5 & 3 & 2 & 4 & 4 & 4 & 5 & 3 & 5 & 4 & 4 & 3 & 5 & 5 & 4 & 3 & 3 & 4 & 2 & 0,91 \\
\hline 38 & 2 & 3 & 4 & 1 & 4 & 4 & 3 & 4 & 4 & 5 & 4 & 5 & 4 & 1 & 5 & 5 & 4 & 4 & 4 & 4 & 2 & 1,45 \\
\hline 39 & 3 & 4 & 4 & 3 & 5 & 4 & 4 & 3 & 5 & 5 & 3 & 4 & 4 & 4 & 5 & 4 & 3 & 4 & 4 & 4 & 4 & 0,45 \\
\hline 40 & 3 & 3 & 2 & 3 & 3 & 3 & 3 & 2 & 3 & 1 & 4 & 2 & 3 & 5 & 1 & 2 & 1 & 3 & 3 & 4 & 4 & 1,09 \\
\hline 41 & 2 & 4 & 4 & 4 & 4 & 4 & 4 & 3 & 5 & 5 & 1 & 4 & 4 & 4 & 5 & 5 & 4 & 4 & 4 & 3 & 4 & 0,93 \\
\hline 42 & 1 & 3 & 4 & 3 & 4 & 2 & 2 & 3 & 4 & 3 & 2 & 3 & 3 & 2 & 5 & 3 & 1 & 3 & 5 & 3 & 3 & 1,15 \\
\hline 43 & 2 & 2 & 2 & 2 & 4 & 1 & 2 & 2 & 1 & 2 & 5 & 2 & 2 & 2 & 1 & 3 & 2 & 3 & 2 & 3 & 2 & 0,89 \\
\hline 44 & 3 & 3 & 4 & 4 & 2 & 4 & 3 & 4 & 5 & 5 & 4 & 5 & 4 & 4 & 5 & 4 & 3 & 2 & 5 & 4 & 4 & 0,83 \\
\hline 45 & 4 & 4 & 4 & 5 & 4 & 5 & 5 & 4 & 5 & 5 & 3 & 5 & 4 & 5 & 5 & 5 & 5 & 4 & 4 & 5 & 5 & 0,36 \\
\hline \multirow[t]{2}{*}{ SUMÍ } & 151 & 147 & 173 & 154 & 173 & 179 & 169 & 148 & 182 & 162 & 163 & 157 & 167 & 175 & 169 & 182 & 182 & 142 & 162 & 153 & 175 & 37,80 \\
\hline & & & & & & & & & & & & & & & & & & & & VAR & 156 & \\
\hline
\end{tabular}

*Para los 21 estudiantes, los valores corresponden a los asignados para cada opción de la 